\title{
Scalar Polynomial Singularities in Power-Law Spacetimes
}

\author{
Kayll Lake* \\ Department of Physics and Department of Mathematics and Statistics, \\ Queen's University, Kingston, Ontario, Canada, KYL $3 N 6$
}

(Dated: September 5, 2018)

\begin{abstract}
Recently, Helliwell and Konkowski (gr-qc/0701149) have examined the quantum "healing" of some classical singularities in certain power-law spacetimes. Here I further examine classical properties of these spacetimes and show that some of them contain naked strong curvature singularities.
\end{abstract}

\section{POWER-LAW SPACETIMES}

The purpose of this study is to examine scalar polynomial singularities associated with power-law spacetimes that can be given in the form

$$
d s^{2}=r^{\alpha} d t^{2}-r^{\beta} d r^{2}-\frac{r^{\gamma}}{C^{2}} d \theta^{2}-r^{\delta}(d z+A d \theta)^{2},
$$

where $\alpha, \beta, \gamma$ and $\delta$ are (real) constants and $A$ and $C$ are functions of $\theta$ only. Helliwell and Konkowski [1] have recently considered the quantum "healing" of classical singularities in the spacetimes (10) (for $C$ constant and $A=0$ ). An elementary transformation in $r$ can be used to simplify (1) into two cases and in the notation of Helliwell and Konkowski these are; $\alpha=\beta$ for $\alpha \neq \beta+2$ (Type I) and $\alpha=\beta+2$ (Type II). The present discussion is organized as follows: first we locate all polynomial singularities in these spacetimes, then we examine the affine distance to the singularities along timelike and null geodesics. Further, we examine details in the $t-r$ subspaces, including the construction of double-null coordinates and finally the focusing conditions associated with the singularities.

\section{SINGULARITIES IN TYPE I}

Consider the Newman-Penrose tetrad [2]

$$
\begin{gathered}
l_{a}=r^{\delta}\left[\sqrt{r^{\beta-\delta}}, 0,-A,-1\right], \\
n_{a}=\frac{1}{2}\left[\sqrt{r^{\beta-\delta}}, 0, A, 1\right], \\
m_{a}=\frac{1}{\sqrt{2}}\left[0,-\sqrt{r^{\beta}},-\frac{i \sqrt{r^{\gamma}}}{C}, 0\right]
\end{gathered}
$$

and

$$
\bar{m}_{a}=\frac{1}{\sqrt{2}}\left[0,-\sqrt{r^{\beta}}, \frac{i \sqrt{r^{\gamma}}}{C}, 0\right] .
$$

This tetrad generates the Type I spacetime

$$
d s^{2}=r^{\beta}\left(d t^{2}-d r^{2}\right)-\frac{r^{\gamma}}{C^{2}} d \theta^{2}-r^{\delta}(d z+A d \theta)^{2} .
$$

The non-vanishing tetrad components of the trace-free Ricci tensor are given by

$$
\begin{gathered}
\Phi 00=\frac{r^{\delta}(\delta+\gamma-2)(\beta-\delta)}{8 r^{\beta} r^{2}}, \\
\Phi 02=\frac{\beta \gamma-\delta^{2}+\delta \gamma+\beta \delta+2 \beta+2 \delta}{16 r^{\beta} r^{2}},
\end{gathered}
$$




$$
\Phi 11=\frac{-\gamma^{2}+\beta \gamma+\beta \delta+2 \gamma}{16 r^{\beta} r^{2}}
$$

and

$$
\Phi 22=\frac{(\beta-\delta)(\delta+\gamma-2)}{32 r^{\beta} r^{\delta} r^{2}}
$$

The non-vanishing tetrad components of the Weyl tensor are given by

$$
\begin{gathered}
\Psi 0=-\frac{r^{\delta}(-\delta+\gamma+2)(\beta-\delta)}{8 r^{\beta} r^{2}}, \\
\Psi 2=-\frac{-3 \beta \delta+\delta^{2}+3 \beta \gamma+\delta \gamma-2 \gamma^{2}+4 \gamma-2 \beta-2 \delta}{48 r^{\beta} r^{2}}
\end{gathered}
$$

and

$$
\Psi 4=-\frac{(-\delta+\gamma+2)(\beta-\delta)}{32 r^{\beta} r^{\delta} r^{2}}
$$

Finally, the Ricci scalar is given by

$$
R=-\frac{-\delta \gamma-\delta^{2}+2 \delta+2 \gamma+2 \beta-\gamma^{2}}{2 r^{\beta} r^{2}}
$$

In general these spacetimes are of Petrov type I but reduce to Petrov type D (and then O) for the following specializations: $\gamma=\delta(\beta=\delta), \gamma=\delta+2(\beta=\delta), \gamma=\beta(\beta=\delta, \beta=\delta-2), \gamma=2 \beta+2-\delta(\beta=\delta, \beta=\delta-2)$.

The tetrad components of the trace-free Ricci and Weyl tensors vanish identically in four cases: (i) $(\beta=\gamma=\delta=0)$, (ii) $(\beta=\delta=0, \gamma=2)$, (iii) $(\beta=\gamma=0, \delta=2)$ and (iv) $(\beta=-2, \delta=\gamma=-2)$. In cases (i), (ii) and (iii) the spacetime is flat. In case (iv) the Ricci scalar reduces to 12 .

Invariants of any spacetime consist of the Ricci scalar, the invariant of lowest degree, and invariants of higher degree constructed from appropriate products of the tetrad components of the trace-free Ricci and Weyl tensors [3] [4]. From (14) it follows that $R$ diverges like $1 / r^{\beta} r^{2}$ as $r \rightarrow 0$ unless

$$
\beta=\gamma^{2} / 2-\gamma+\delta^{2} / 2-\delta+\delta \gamma / 2
$$

Substitution of $\beta$ from (15) into the Ricci invariant of next degree shows that the invariant diverges like $\left(1 / r^{\beta} r^{2}\right)^{2}$ as $r \rightarrow 0$ unless [5]

$$
(\delta-2+\gamma)^{2}\left(\delta^{2}-2 \delta+4+\gamma \delta-2 \gamma+\gamma^{2}\right)=0
$$

Substitution of $\beta$ from (15) and $\delta=2-\gamma$ from (16) into the first Weyl invariant shows that this invariant diverges like $\left(1 / r^{\beta} r^{2}\right)^{2}$ as $r \rightarrow 0$ unless

$$
\gamma^{2}(\gamma-2)^{2}=0
$$

The case $\gamma=0$ gives $\delta=2$ and $\beta=0$, that is, the flat case (iii). The case $\gamma=2$ gives $\delta=0$ and $\beta=0$, that is the flat case (ii). Substitution of $\beta$ from (15) and $\delta^{2}-2 \delta+4+\gamma \delta-2 \gamma+\gamma^{2}=0$ from (16) into the first Weyl invariant shows that this invariant diverges as $\left(1 / r^{\beta} r^{2}\right)^{2}$ as $r \rightarrow 0$ for all real $\gamma$ and $\delta$. Finally, note that both factors in (16) cannot vanish simultaneously for real $\gamma$ and $\delta$.

It follows that for Type I power-law spacetimes, except for the four cases (i) through (iv), the spacetimes contain a scalar polynomial singularity at $r=0$ for all $\beta+2>0$. 


\section{SINGULARITIES IN TYPE II}

Now consider the Newman-Penrose tetrad consisting of (2) and (3), both with $\beta$ replaced by $\beta+2$, along with (4) and (5) unchanged. This tetrad generates the Type II spacetime

$$
d s^{2}=r^{\beta+2}\left(d t^{2}-\frac{d r^{2}}{r^{2}}\right)-\frac{r^{\gamma}}{C^{2}} d \theta^{2}-r^{\delta}(d z+A d \theta)^{2} .
$$

The non-vanishing tetrad components of the trace-free Ricci tensor are now given by

$$
\begin{gathered}
\Phi 00=\frac{r^{\delta}(\beta-\delta+2)(\delta+\gamma)}{8 r^{\beta} r^{2}}, \\
\Phi 02=\frac{\beta \gamma-\delta^{2}+\delta \gamma+\beta \delta+2 \gamma+2 \delta}{16 r^{\beta} r^{2}}, \\
\Phi 11=\frac{-\gamma^{2}+\beta \gamma+\beta \delta+2 \gamma+2 \delta}{16 r^{\beta} r^{2}}
\end{gathered}
$$

and

$$
\Phi 22=\frac{(\beta-\delta+2)(\delta+\gamma)}{32 r^{\beta} r^{\delta} r^{2}} .
$$

The non-vanishing tetrad components of the Weyl tensor are now given by

$$
\begin{gathered}
\Psi 0=-\frac{r^{\delta}(-\delta+\beta+2)(\gamma-\delta)}{8 r^{\beta} r^{2}}, \\
\Psi 2=-\frac{(\gamma-\delta)(-\delta-2 \gamma+6+3 \beta)}{48 r^{\beta} r^{2}},
\end{gathered}
$$

and

$$
\Psi 4=-\frac{(-\delta+\beta+2)(\gamma-\delta)}{32 r^{\beta} r^{\delta} r^{2}}
$$

Finally, the Ricci scalar is now given by

$$
R=\frac{\delta^{2}+\delta \gamma+\gamma^{2}}{2 r^{\beta} r^{2}}
$$

In general these spacetimes are of Petrov type I but reduce to Petrov type $\mathrm{O}$ for $\gamma=\delta$, and Petrov type $\mathrm{D}$ (and then $\mathrm{O})$ for the following specializations: $\gamma=\beta+2(\beta=\delta-2), \gamma=2 \beta+4-\delta(\beta=\delta-2)$.

The tetrad components of the trace-free Ricci and Weyl tensors now vanish identically only for $\delta=\gamma=0$ in which case the spacetime is flat. Except in this case the Ricci scalar itself diverges like $1 / r^{\beta} r^{2}$ as $r \rightarrow 0$.

It follows that for Type II power-law spacetimes, except for the flat case $\delta=\gamma=0$, the spacetimes contain a scalar polynomial singularity at $r=0$ for all $\beta+2>0$.

\section{AFFINE DISTANCE TO THE SINGULARITIES}

Geodesics of the spacetimes (1) satisfy

$$
r^{\beta} \dot{r}^{2}=\frac{c_{1}^{2}}{r^{\alpha}}-\frac{r^{\gamma} \dot{\theta}^{2}}{C^{2}}-\frac{c_{2}^{2}}{r^{\delta}}-2 \mathcal{L}
$$


where $2 \mathcal{L}=0$ in the null case and $2 \mathcal{L}=1$ in the timelike case, $c_{1}$ and $c_{2}$ are constants of the motion and ${ }^{\circ} \equiv d / d \lambda$ where $\lambda$ is an affine parameter. It follows from (27) that

$$
\lambda_{*}-\lambda_{0} \geq \int_{0}^{r_{*}} r^{(\alpha+\beta) / 2} d r
$$

where $\lambda_{*}$ and $r_{*}$ are finite and non-zero. As a result, the singularities at $r=0$ are at finite affine distance if and only if

$$
\alpha+\beta+2>0 .
$$

Whereas condition (29) offers no further restriction on $\beta$ for singularities in Type II spacetimes (we again have $\beta+2>0$ ), for Type I spacetimes we obtain the refined condition $\beta+1>0$ for singularities, assuming, as usual, that they must be at a finite affine distance.

\section{THE $t-r$ SUBSPACES $\Sigma$}

In what follows we examine in further detail the $t-r$ subspaces (designated by $\Sigma$ ) of the spacetimes (1). Since we are primarily interested in the structure of the singularities at $r=0$ we first explore the conditions under which null geodesics can terminate there. Now transforming from $\theta$ to $\tilde{\theta}$ where $d \theta / C(\theta)=d \tilde{\theta}$ we have a constant of the motion $c_{3}$ and the null geodesic equation can be given as

$$
r^{\beta} \dot{r}^{2}=\frac{1}{r^{\alpha}}-\frac{c_{2}^{2}}{r^{\delta}}-\frac{c_{3}^{2}}{r^{\gamma}}
$$

where, without loss in generality, we have set $c_{1}^{2}=1$. There are four cases of interest: (i) If $c_{2}=c_{3}=0$ then clearly there are no turning points $\dot{r}=0$ at finite $r$. (ii) If $c_{2}=0, c_{3} \neq 0$ then there is a minimum $r$ at $r_{0}>0$ for $\alpha \geq \gamma$. (iii) If $c_{2}=0, c_{3} \neq 0$ then there is a minimum $r$ at $r_{0}>0$ for $\alpha \geq \delta$. (iv) If $c_{2} \neq 0$ and $c_{3} \neq 0$ then there is a minimum $r$ at $r_{0}$ where

$$
\frac{1}{r_{0}^{\alpha}}=\frac{c_{2}^{2}}{r_{0}^{\delta}}+\frac{c_{3}^{3}}{r_{0}^{\gamma}}
$$

as long as the constants of the motion satisfy

$$
c_{2}^{2}(\delta-\gamma) \leq(\alpha-\gamma) r_{0}^{\delta-\alpha}
$$

and

$$
c_{3}^{2}(\gamma-\delta) \leq(\alpha-\delta) r_{0}^{\gamma-\alpha} .
$$

If there are minima in $r$ along null geodesics then a discussion in the $t-r$ subspace of the spacetimes (1) below the minima is adequate for these geodesics in the sense that they do not populate that region of the $t-r$ subspace. However, since there are geodesics for which $c_{2}$ and $c_{3}$ do not vanish simultaneously and which terminate at $r=0$, the $t-r$ subspaces are not totally geodesic and discussions restricted to these subspaces are not complete. However, an examination of these subspaces is, though incomplete, very instructive. We now construct double- null coordinates and examine focusing conditions.

\section{A. Double Null Coordinates}

It is clear from (6) and (18) that the trajectories $r=r_{0}=$ constant $>0$ are timelike for $r>0$. However, the nature of the singularities at $r=0$ is not clear. Here we construct double-null coordinates to clarify the nature of the singularities. By double-null coordinates we mean coordinates which label affinely parameterized null geodesics of the full spacetime. The associated spacetime diagrams that we draw are, however, restricted to the $t-r$ subspaces. 


\section{Type $I$}

On $\Sigma$ we have

$$
d s_{\Sigma}^{2}=r^{\beta}(d t-d r)(d t+d r)
$$

Introducing null coordinates $u$ and $v$ where $u=$ constant along $d t=d r$ and $v=$ constant along $d t=-d r$ and writing $r=f(u, v)$ and $t=g(u, v)$ it follows that

$$
f(u, v)=g(u, v)+F(u)=-g(u, v)+H(v) .
$$

With the choices

$$
F(u)=2 u, \quad H(v)=2 v,
$$

we have

$$
r=u+v, \quad t=v-u,
$$

so that

$$
d s_{\Sigma}^{2}=-4(u+v)^{\beta} d u d v
$$

where we have orientated the future so that $d v>0$ but $d u<0$. We have the following tangents to null geodesics in the full spacetime (6) subject to the transformations (37): along $v=v_{0}=$ constant

$$
k^{a}=-\frac{\delta_{u}^{a}}{\left(u+v_{0}\right)^{\beta}}
$$

so that $k^{b} \nabla_{b} k^{a}=0$ and along $u=u_{0}=$ constant

$$
l^{a}=\frac{\delta_{v}^{a}}{\left(v+u_{0}\right)^{\beta}}
$$

so that $l^{b} \nabla_{b} l^{a}=0$. As a result, the singularities at $r=0$ are at finite affine distance only for $\beta+1>0$ as discussed above. The trajectories $r=$ constant are straight vertical lines and timelike throughout the $u-v$ diagram. Trajectories of constant $t$ are straight horizontal lines and spacelike throughout the $u-v$ diagram. A diagram is shown below in Figure1

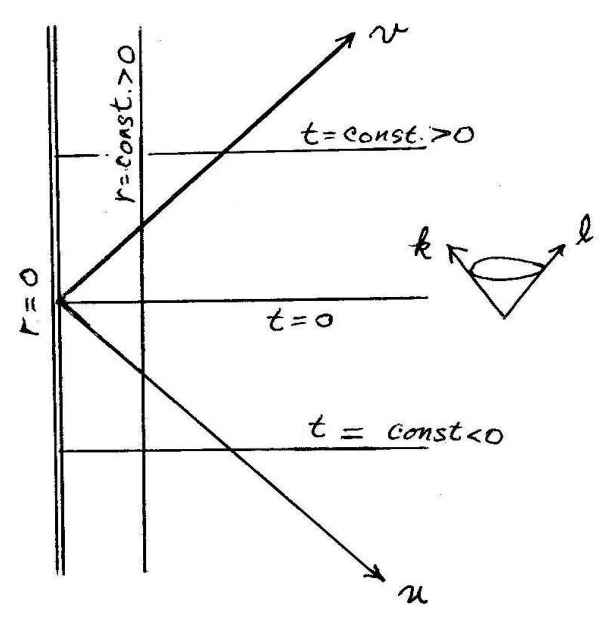

FIG. 1: Representation of the $t-r$ subspace for Type I power-law spacetimes in the double-null coordinates $u$ and $v$. The singularity at $r=0$ is timelike. 


\section{Type II}

On $\Sigma$ we now have

$$
d s_{\Sigma}^{2}=r^{\beta+2}\left(d t-\frac{d r}{r}\right)\left(d t+\frac{d r}{r}\right)
$$

Introducing null coordinates $u$ and $v$ where now $u=$ constant along $d t=\frac{d r}{r}$ and $v=$ constant along $d t=-\frac{d r}{r}$ and again writing $r=f(u, v)$ and $t=g(u, v)$ it now follows that

$$
f(u, v)=F(u) e^{g(u, v)}=H(v) e^{-g(u, v)} .
$$

With the choices

$$
F(u)=u^{2}, \quad H(v)=v^{2},
$$

so that $u$ and $v$ are not simultaneously zero, we have

$$
r=u v, \quad t=\ln \left(\frac{v}{u}\right),
$$

so that

$$
d s_{\Sigma}^{2}=-4(u v)^{\beta+1} d u d v
$$

where again we have orientated the future so that $d v>0$ but $d u<0$. We now have tangents to null geodesics in the full spacetime (18) subject to the transformations (44)

$$
k^{a}=-\frac{\delta_{u}^{a}}{u^{\beta+1}}
$$

so that $k^{b} \nabla_{b} k^{a}=0$ and

$$
l^{a}=\frac{\delta_{v}^{a}}{v^{\beta+1}}
$$

so that $l^{b} \nabla_{b} l^{a}=0$. As a result, the singularities at $r=0$ are at finite affine distance only for $\beta+2>0$ also as shown above. The surfaces $r=$ constant $>0$ are now hyperbolae in the $u-v$ diagram and timelike. They become the degenerate null hyperbola for $r=0$. Trajectories of constant $t$ are straight lines through the origin $u=v=0$ which is a point of internal infinity. A diagram is shown below in Figure2

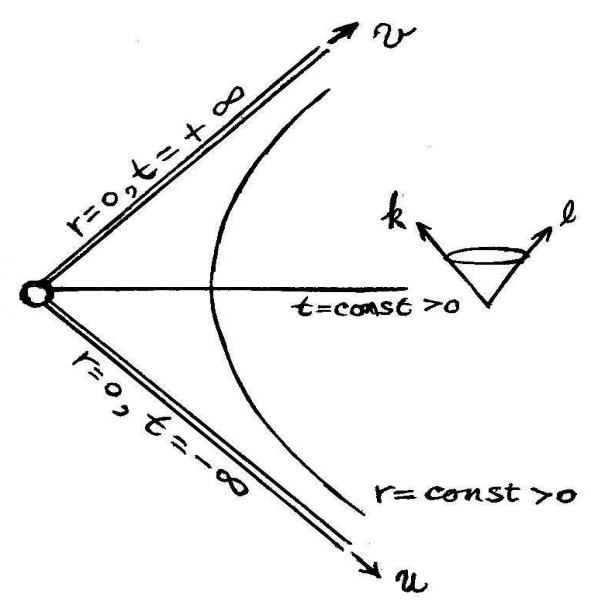

FIG. 2: Representation of the $t-r$ subspace for Type II power-law spacetimes in the double-null coordinates $u$ and $v$. The singularity at $r=0$ is either past or future null. The origin $u=v=0$ is a point of internal infinity. 


\section{B. Focusing conditions}

In this section we consider a unified treatment of both types of spacetime. The vector

$$
k^{a}=\left(1 / r^{\alpha}, \pm 1 / r^{(\alpha+\beta) / 2}, 0,0\right)
$$

is tangent to a class of null geodesics of the spacetimes (1) and we use (48) to examine focusing conditions in the $t-r$ subspaces. Define

$$
\Psi \equiv \lambda^{n} R_{a b} k^{a} k^{b}
$$

where $R_{a b}$ is the Ricci tensor. We find

$$
\Psi=r^{(n / 2-1)(\alpha+\beta+2)} \Delta
$$

where

$$
\Delta=2 \beta(\gamma+\delta)+\gamma(2-\gamma)+\delta(2-\delta) \equiv \Delta_{I}
$$

for Type I and

$$
\Delta=2 \beta(\gamma+\delta)+\gamma(4-\gamma)+\delta(4-\delta) \equiv \Delta_{I I}
$$

for Type II. Clearly $\Psi=0 \forall n$ with $\Delta=0$. For $\Delta \neq 0, \Psi \rightarrow 0$ as $r \rightarrow 0$ for $n>2$ and $|\Psi| \rightarrow \infty$ as $r \rightarrow 0$ for $n<2$. For $n=2, \Psi=\Delta$ and for $\Delta>0$ the singularities at $r=0$ satisfy the strong curvature condition [ 6 ].

\section{CONCLUSIONS}

Type I spacetimes can have strong curvature timelike naked singularities at $r=0$ for $\beta+1>0$ and $\Delta_{I}>0$. Type II spacetimes can have strong curvature past-null naked singularities at $r=0$ for $\beta+2>0$ and $\Delta_{I I}>0$.

\section{Acknowledgments}

It is a pleasure to thank Thomas Helliwell and Deborah Konkowski for comments and in particular for suggesting a clarification of the Type II singularities. This work was supported by a grant from the Natural Sciences and Engineering Research Council of Canada and was made possible by use of GRTensorII [7].

[*] Electronic Address: lake@astro.queensu.ca

[1] T. M. Helliwell and D. A. Konkowski gr-qc/0701149

[2] See, for example, H. Stephani, D. Kramer, M. MacCallum, C. Hoenselaers and E. Herlt, Exact Solutions of Einstein's Field Equations (Cambridge University Press, Cambridge, 2003) chapter 7.

[3] See, for example, H. Stephani et al. [2] chapter 9.

[4] The Kretschmann scalar (the full contraction of the Riemann tensor) is not used here since it is a linear combination of the square of the Ricci scalar, the first Ricci invarinat and the real component of the first Weyl invariant.

[5] We exclude factors which have no non-vanishing real roots.

[6] See, for example, C. J. S. Clarke, The Analysis of Space-Time Singularities (Cambridge University Press, Cambridge, 1993).

[7] This is a package which runs within Maple. It is entirely distinct from packages distributed with Maple and must be obtained independently. The GRTensorII software and documentation is distributed freely on the World-Wide-Web from the address http://grtensor.org 